\title{
Inflow occlusion pulmonary embolectomy in the modern era of cardiac surgery
}

\author{
Joseph L. Bobadilla, MD, Christopher H. Wigfield, MD, \\ and Paramjeet S. Chopra, MD, Madison, Wis
}

$\mathrm{P}$ ulmonary embolism (PE) continues to be a significant cause of morbidity and mortality. It is estimated that nearly 200,000 to 300,000 PEs are diagnosed annually in the United States: approximately 1 per 1000 persons per year. Additionally, PE is associated with 50,000 to 100,000 deaths annually. ${ }^{1}$ Operative intervention, although rarely performed, is indicated in patients with massive embolism resulting in hemodynamic instability. Massive embolism is defined as a PE associated with systolic pressures less than $90 \mathrm{~mm}$ $\mathrm{Hg}$ or a $40 \mathrm{~mm} \mathrm{Hg}$ or greater decrease from baseline for longer than 15 minutes. ${ }^{2}$ Patients with such embolic burden often rapidly progress to refractory hypotension and subsequent cardiopulmonary arrest. This patient population may benefit from surgical embolectomy.

\section{Clinical Summary}

We present a case of a 48-year-old black woman who presented with severe cerebral hemorrhage. She subsequently developed a pulmonary embolus and experienced worsening hypoxemia and a precipitous decrease in mean arterial pressure $(>40 \mathrm{~mm} \mathrm{Hg})$. There was no identifiable septic or primary cardiac cause for this abrupt change. Echocardiographic evaluation showed signs of right heart strain and akinesis of the mid free right ventricular wall, with preserved apical motion (positive McConnell sign). Computed tomographic angiogram showed significant embolic burden, with a large saddle embolism (Figure 1). In view of the contraindications to thrombolytic/anticoagulation therapy, refractory hypotension despite maximal vasopressors, and worsening hypoxemia, urgent surgical embolectomy was considered.

Inflow occlusion pulmonary embolectomy was performed, guided by intraoperative echocardiographic monitoring. A me-

\footnotetext{
From the Department of Cardiothoracic Surgery, University of Wisconsin, Hospital and Clinics, Madison, Wis.

Received for publication June 16, 2005; accepted for publication Aug 8, 2005.

Address for reprints: Paramjeet S. Chopra, MD, University Hospital, Department of Cardiothoracic Surgery, 600 Highland Ave, Madison, WI 53792-3236 (E-mail: chopra@ surgery.wisc.edu).

J Thorac Cardiovasc Surg 2006;131:484-6

$0022-5223 / \$ 32.00$

Copyright $\odot 2006$ by The American Association for Thoracic Surgery

doi:10.1016/j.jtcvs.2005.08.041
}

dian sternotomy was

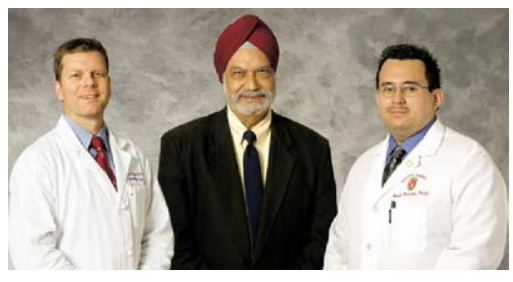

Drs C. Wigfield, P. Chopra, and J. Bobadilla (left to right)

performed, and the pulmonary artery and aorta were isolated independently. Traction sutures were placed in the proximal/distal pulmonary artery, as were 4 lateral stay sutures. Tapes were passed around the superior and inferior vena cava and secured with rubber tourniquets (Figure 2). During a 7-minute period of preoxygenation and the administration of steroids, the core temperature was allowed to drift to $32^{\circ} \mathrm{C}$. The vena caval tapes were then snared. An arteriotomy was made between the previously placed traction sutures in the longitudinal axis of the pulmonary artery. The emboli were then retrieved by vigorous suction extraction and ring forceps.

The patient was extubated on postoperative day 3. Sixty days after the initial cerebral hemorrhage, she was doing very well (conversing and ambulating with a walker) and had returned home to live with her family.

\section{Comment}

Currently, surgical pulmonary embolectomy is reserved only for patients who demonstrate profound cardiovascular collapse as a result of their embolic burden. Dating back to the early 1900s, sporadic case reports have documented attempts at open embolectomy. The earliest of these dates back to 1908, with Trendelenburg's classic description. ${ }^{3}$ Pulmonary artery exposure was achieved via a $\mathrm{T}$-shaped incision over the second costal margin immediately to the left of the sternum. A Sound was then passed around the aorta and pulmonary artery, the pulmonary artery was incised, and the clot was extracted with forceps and suction. It was not until 1924 that the first successful pulmonary embolectomy was performed by Kirschner, ${ }^{4}$ Trendelenburg's assistant.

A review by Gibbon $^{5}$ in 1937 estimated that some 142 persons had undergone the Trendelenburg embolectomy, and only 9 had survived. Investigation into open cardiac repairs and the use of hypothermia was pioneered by Swan and colleagues ${ }^{6}$ and Allison and associates ${ }^{7}$ in the early 1950s and 1960s. ${ }^{6,7}$ The modern equivalent has evolved from early studies in inflow occlusion and intra-arterial approaches to pulmonary valvotomy. ${ }^{8-11}$

Profound cardiac and neurologic complications manifest if occlusion times increase past 3 minutes. Cooling blankets, hypothermia, and steroids have helped reduce these devastating complications, but profound postoperative deficits are still common.

Since the advent of cardiopulmonary bypass (CPB) in the early 1960s, most embolectomies have been performed with patients on pump. The first such operations were performed by Cooley and colleagues $^{12}$ in 1961 and Sharp ${ }^{13}$ in 1962. CPB-assisted embolec- 

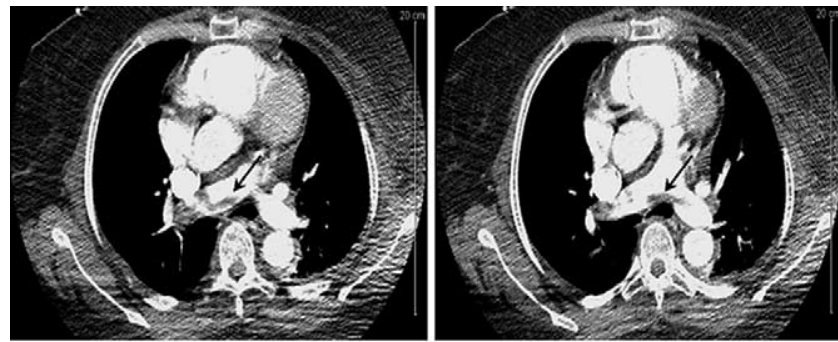

Figure 1. Computed tomographic angiogram (chest) with helical acquisition and $1.25-\mathrm{mm}$ collimation. There is evidence of massive pulmonary embolism (arrows), including a saddle embolus extending into both main pulmonary arteries. The clot burden is greatest in the right lower lobe, with occlusive-appearing clot seen in all segmental arteries. The central pulmonary arteries are enlarged and consistent with acute pulmonary arterial hypertension.
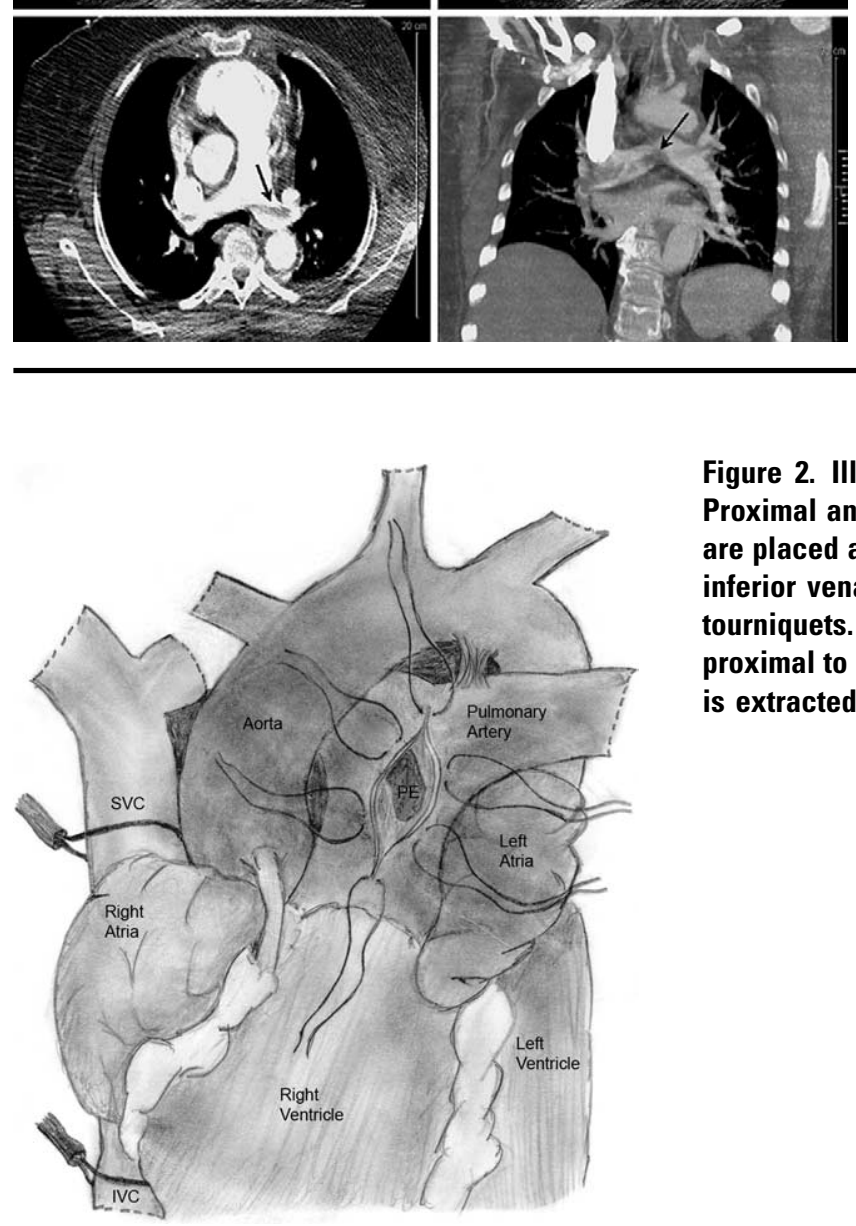

Figure 2. Illustration showing exposure for the inflow occlusion embolectomy. Proximal and distal pulmonary artery stay sutures are placed, and 4 stay sutures are placed adjacent to the planned arteriotomy site. Tapes are passed around the inferior vena cava (IVC) and superior vena cava (SVC) and secured with rubber tourniquets. Incision is made between the latter stay sutures extending from the proximal to distal anchor sutures. Aggressive suction is applied, and the embolus is extracted with suction or forceps delivery.

tomies have historically shown improved survival and significantly fewer postoperative neurologic morbidities.

Even in modern times, CPB is not always readily available; furthermore, there exists a discrete population in which the risks of CPB far outweigh the potential benefits of its use in embolectomy. It is in these clinical scenarios where inflow occlusion embolectomy provides a reasonable alternative to $\mathrm{CPB}$-assisted embolectomy. With the judicious application of brief episodes of venous inflow stasis of up to 3 minutes, pulmonary embolectomy is perfectly feasible without the use of CPB. The limitation of this approach is clearly related to cerebral and systemic malperfusion; therefore, the procedure must be performed with a concerted effort and full awareness of its associated mortality and morbidity as a surgical salvage treatment when no other surgical option is available. We report this as another valuable tool in the surgeon's armamentarium when dealing with massive PE.

\section{References}

1. Horlander KT, Mannino DM, Leeper KV. Pulmonary embolism mortality in the United States, 1979-1998: an analysis using multiple-cause mortality data. Arch Intern Med. 2003;163:1711-7.

2. Task Force on Pulmonary Embolism, European Society of Cardiology. Guidelines on diagnosis and management of acute pulmonary embolism. Eur Heart J. 2000;21:1301-36.

3. Trendelenburg F. Zur Operation der Embolie der Lungenarterie. Dtsch Med Wochenschr. 1908;34:1172. 
4. Kirschner M. Ein durch die Trendelenburgische Operation geheiter Fall von Embolie der Art. Pulmonalis. Arch Klin Chir. 1924;133:312.

5. Gibbon JH. Artificial maintenance of circulation during experimental occlusion of pulmonary artery. Arch Surg. 1937;34:1105

6. Swan H, Zeavin I, Blount SG Jr, Virtue RW. Surgery by direct vision in the open heart during hypothermia. JAMA. 1953;153:1081.

7. Allison PR, Dunnill MS, Marshall R. Pulmonary embolism. Thorax. 1960;15:273.

8. Berkas EM, Ferguson DJ, Garamella JJ, et al. Studies on techniques permitting prolonged cardiac venous inflow stasis. Surg Forum. 1953; 4:59-62.
9. Vossschulte K, Stiller H, Eisenreich F. Emergency embolectomy by the transsternal approach in acute pulmonary embolism. Surgery. 1965;58:317-23

10. Lam CR, Taber RE. Simplified technique for direct vision pulmonary valvotomy. J Thorac Cardiovasc Surg. 1959;38:309-18.

11. Mistrot J, Neal W, Lyons G, et al. Pulmonary valvulotomy under inflow stasis for isolated pulmonary stenosis. Ann Thorac Surg. 1976;21:30-7.

12. Cooley DA, Beall AC, Alexander JK. Acute massive pulmonary embolism. JAMA. 1961;177:283-6.

13. Sharp EH. Pulmonary embolectomy: successful removal of a massive pulmonary embolus with the support of cardiopulmonary bypass: case report. Ann Surg. 1962;156:1.

\section{A primary aortoesophageal fistula due to esophageal carcinoma successfully treated with endoluminal aortic stent grafting}

Yoshifumi Ikeda, MD, Naomi Morita, MD, Hideko Kurihara, MD, Masanori Niimi, MD, and Kota Okinaga, MD, Tokyo, Japan

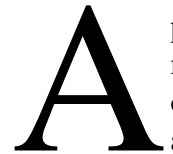

primary aortoenteric fistula is described as the communication between the aorta and the intestine without previous aortic surgery. Aortoesophageal fistula is a relatively rare but life-threatening cause of upper gastrointestinal tract bleeding. A thoracic aortic aneurysm is the most common reported cause, and ulceration of a carcinoma is a rarity. ${ }^{1}$ Although surgeons agree that the treatment of the primary aortoesophageal fistula should be surgical, the mortality is very high. Herein, we report a primary aortoesophageal fistula due to esophageal carcinoma successfully treated by using endoluminal aortic stent grafting.

\section{Clinical Summary}

A 64-year-old man was admitted to our department with persistent midthoracic pain, severe dysphagia, and a weight loss of $8 \mathrm{~kg}$ during the previous 3 months. An upper gastrointestinal endoscopic study revealed a friable tumor $5 \mathrm{~cm}$ in length in the middle thoracic esophagus. The esophageal tumor was diagnosed as a moderately differentiated squamous cell carcinoma by endoscopic biopsy samples. A computed tomographic scan revealed that the tumor invaded into the descending aorta. Therefore, the patient was treated with combination cis-dichlorodiammineplatinum and

From the Department of Surgery, Teikyo University School of Medicine, Tokyo, Japan.

Received for publication July 15, 2005; revisions accepted Aug 26, 2005; accepted for publication Aug 30, 2005.

Address for reprints: Yoshifumi Ikeda, MD, Department of Surgery, Teikyo University School of Medicine, 2-11-1 Kaga, Itabashi-ku, Tokyo, 1738605, Japan (E-mail: yikeda@med.teikyo-u.ac.jp).

J Thorac Cardiovasc Surg 2006;131:487-8

$0022-5223 / \$ 32.00$

Copyright $\odot 2006$ by The American Association for Thoracic Surgery doi:10.1016/j.jtcvs.2005.08.042

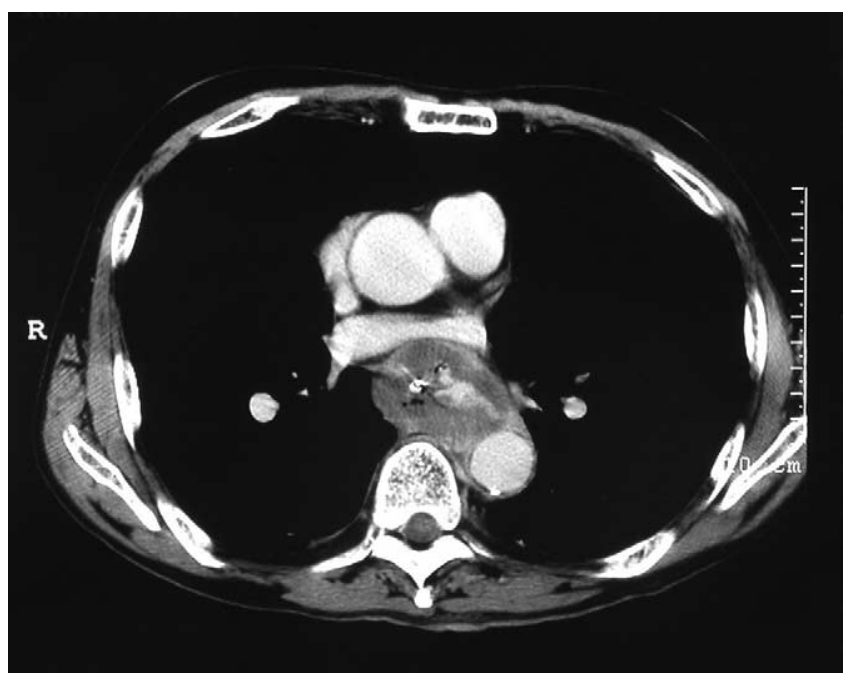

Figure 1. A computed tomographic scan revealed that the tumor invaded into the descending aorta and had a communication between the aorta and the esophagus.

fluorouracil adjuvant chemotherapy. The chemotherapy regimen consisted of $70 \mathrm{mg}$ of cis-dichlorodiammineplatinum on day 1 and $750 \mathrm{mg}$ of fluorouracil on days 1 to 5 , intravenously. Seven days after the chemotherapy, the patient experienced hemorrhagic shock with massive upper intestinal bleeding. After crystalloid transfusion, the patient recovered from the hemorrhagic shock. A computed tomographic scan and aortography were performed. Both tests showed a communication between the aorta $10 \mathrm{~cm}$ distal to the ostium of the left subclavian artery and the esophagus in its middle segment (Figure 1). To control massive upper intestinal bleeding, a stent graft made from a Gianturco Z-stent (William 